\title{
Plasma biasing to control the growth conditions of diamond-like carbon
}

\author{
André Anders ${ }^{1}$, Nitisak Pasaja ${ }^{1,2}$, Sunnie H.N Lim ${ }^{1}$, Tim C. Petersen ${ }^{3}$, Vicki J. Keast ${ }^{3}$ \\ ${ }^{1}$ Lawrence Berkeley National Laboratory, University of California, Berkeley, California \\ 94720, USA. \\ ${ }^{2}$ Physics Department, Faculty of Science, Chiang Mai University, Chiang Mai 50200, \\ Thailand. \\ ${ }^{3}$ Australian Key Centre for Microscopy and Microanalysis, University of Sydney, \\ Sydney, New South Wales 2006, Australia.
}

\begin{abstract}
It is well known that the structure and properties of diamond-like carbon, and in particular the $\mathrm{sp}^{3} / \mathrm{sp}^{2}$ ratio, can be controlled by the energy of the condensing carbon ions or atoms. In many practical cases, the energy of ions arriving at the surface of the growing film is determined by the bias applied to the substrate. The bias causes a sheath to form between substrate and plasma in which the potential difference between plasma potential and surface potential drops. In this contribution, we demonstrate that the same results can be obtained with grounded substrates by shifting the plasma potential. This "plasma biasing" (as opposed to "substrate biasing”) is shown to work well with pulsed cathodic carbon arcs, resulting in tetrahedral amorphous carbon (ta-C) films that are comparable to the films obtained with the conventional substrate bias. To verify the plasma bias approach, ta-C films were deposited by both conventional and plasma bias and characterized by transmission electron microscopy (TEM) and electron energy loss spectrometry (EELS). Detailed data for comparison of these films are provided.
\end{abstract}




\section{Introduction}

It is well known that the bonding structure of diamond-like carbon (DLC), and in particular the $\mathrm{sp}^{3} / \mathrm{sp}^{2}$ ratio, can be controlled and tuned by the energy of the condensing carbon ions or atoms. Other factors, such as substrate temperature, deposition rate, the presence of background gas or growth-assisting argon ions does also play a role but we focus on the effects of the energy of condensing species. In most cases, the energy of ions arriving at the surface of the growing film is determined by a negative bias applied to the substrate. The bias causes a sheath to form between substrate and plasma in which the potential difference between plasma potential and surface potential drops. Electrons are repelled and returned to the plasma, while ions are accelerated towards the substrate surface.

Often, it is advantageous to use pulsed bias, as opposed to dc (direct current) bias, in order to avoid the charge-up of the rather insulating films. Charge-up of the film surface reduces ion energy in an uncontrolled manner, and it may lead to breakdown of film and damage by arcing on the substrate. When the bias is off, the sheath collapses and allows electrons to reach the surface, compensating the excess positive charge [1].

In this contribution, we demonstrate that the same effect on the ion energy can be obtained when the substrate is grounded and the plasma is positively biased. Shifting the plasma potential may be called "plasma biasing," as opposed to "substrate biasing."

Generally, plasma biasing is not new but commonly not practiced for two reasons. Firstly, it requires the components of the power supply components to be suitably designed, with the circuit reference ground-free, allowing it to be shifted with respect to ground. Secondly, a positively biased plasma forms a sheath to all grounded 
components, such as chamber walls, shields, etc., and this may cause unwanted side effects such as wall sputtering and contamination of the substrate surface. Additionally, at high bias and in the presence of dense plasma, arc spots may ignite on the grounded components (“arcing”). However, with careful system design using shields etc. one can avoid these issues.

In this contribution it is shown that plasma biasing works well with pulsed cathodic carbon arcs, resulting in tetrahedral amorphous carbon (ta-C) films that are comparable to films obtained with the more conventional substrate bias. Samples of ta-C films were made by plasma biasing and conventional biasing were characterized by transmission electron microscopy (TEM) and electron energy loss spectrometry (EELS).

\section{Experimental}

For the deposition of thin ta-C films we used a miniature pulsed cathodic arc source of the "minigun" type [2]. Such source has a rod cathode of $6.25 \mathrm{~mm}$ diameter (1/4 inch) and about $30 \mathrm{~mm}$ length. All but the front face is enclosed by a ceramic tube, and therefore cathode spots can only exist on the rod's small front face. The location of plasma production is thereby well defined. The anode is an annular cylinder of about 25 mm inner diameter. Repetitively pulsed arc ignition is facilitated by the "triggerless" or “self-trigger" method, i.e. by a current driven between anode and cathode via a conducting coating on the cathode-enclosing ceramic [3].

The plasma flow entered an open-coil, $90^{\circ}$-bent macroparticle filter, which was electrically in series with the arc. In this way, detrimental "macroparticles" are effectively filtered from the plasma and high-quality films can be obtained [4]. The distance from filter exit to substrate was $15 \mathrm{~cm}$. 
In a common setup, Fig. 1(a), the arc anode is grounded and the filter entrance turn is connected to the anode, while the filter exit turn is connected to the positive terminal of the power supply [5]. The plasma exiting this kind of system has a plasma potential not far from the ground potential. The substrate is negatively pulsed biased, using the anode (ground) as a reference potential. For pulsed bias we selected bursts with $10 \mu$ s on and $30 \mu$ s off, hence the bias duty cycle was $25 \%$. The bias supply was a tubeswitched pulser (Cober Inc., max $2.5 \mathrm{kV}$ ). Each arc pulse was about $1 \mathrm{~ms}$ long; the arc pulse repetition rate was low, only 0.7 p.p.s., and therefore the substrate temperature remained very close to room temperature. The base pressure of the cryogenically pumped chamber was about $10^{-4} \mathrm{~Pa}$; no working gas was used during processing.

It is well known that ta-C films can be deposited with such configuration, and that film properties such as $\mathrm{sp}^{3} / \mathrm{sp}^{2}$ ratio, intrinsic stress, hardness, etc. can be controlled with the bias. Therefore, we do not report here about extensive sets of samples.

The interesting work was done when modifying the setup for plasma biasing, as shown in Fig. 1(b). Fig. 2 shows the specific circuitry that we employed to shift the plasma potential.

Fig. 3 shows the actual arc current, plasma bias voltage, and the bias current needed to drive the bias voltage. The arc current was measured with a 0.01V/A current transformer (Pearson model 1114), the plasma bias voltage was monitored by a 1000:1 compensated voltage probe (Tektronix model P6015A), and the bias current was determined by a current transformer with 0.1 V/A (Pearson model 110).

Six samples of ta-C thin films were investigated. The deposition conditions were selected in such a way that they allowed us to compare ta-C film properties obtained by 
plasma biasing versus conventional substrate biasing. We also included bias conditions with the substrate grounded and at floating potential. All films on silicon substrates showed the interference colors that are typical for non-uniform transparent films on a reflecting substrate. At the same time, salt crystals were used as substrates for the electron microscopy studies.

The thin films were characterized by electron energy loss spectroscopy (EELS) and by transmission electron microscopy (TEM) using a $120 \mathrm{kV} \mathrm{LaB}{ }_{6}$ Phillips CM120 TEM equipped with a Gatan Imaging Filter (GIF). TEM specimens were prepared by dissolving the salt substrates in distilled water. Low loss and carbon k-edge EEL spectra were collected from between six and eight different regions of each sample, using a dispersion of $0.1 \mathrm{eV}$ per channel. Each of these spectra was then analyzed individually to give an estimate of the variation in results. EELS analysis of a highly graphitic ultramicrotomed glassy carbon specimen was also conducted to provide a spectral reference of a solid containing practically complete $\mathrm{sp}^{2}$ bonding [6]. As a consistency check, EELS data of one of the specimen was also collected using a high-resolution Scanning TEM (VGSTEM HB601) equipped with an Enfina spectrometer. Several areas of the specimen were examined with the VGSTEM (using the same dispersion) and were likewise analyzed to give an estimate of uncertainty in the data.

For low-loss EELS of disordered carbon solids, the position of the dominant plasmon peak gives an indication of the specimen density, since the plasmon energy is approximately proportional to the square root of the number of valence electrons per unit volume [7]. The position of this peak is thus commonly used to characterize DLC thin films [8]. For non-hydrogenated amorphous films it has been shown that there is linear 
correlation between the plasmon-inferred density and the $\mathrm{sp}^{3}$ content [9]. Experimental investigations [10] and theoretical considerations of ta-C [9] have shown that it is appropriate to use prescribe an effective mass $m^{*} \cong 0.9 \mathrm{~m}_{\mathrm{e}}$ for the valence electrons when calculating the specimen density from the plasmon peak position, where $m_{\mathrm{e}}$ is the rest mass of the electron. However, for the sake of simplicity, the approximate densities in this paper are quoted using $m^{*}=m_{\mathrm{e}}$. We also approximate the plasmon energies as equal to the maxima in the corresponding peak positions in the EEL spectra.

Prior to the carbon k-edge, there exists a $1 \mathrm{~s} \rightarrow \pi^{*}$ peak which varies in extent, depending upon the amount of $\mathrm{sp}^{2}$ bonding in the specimen. The relative area under this peak in the EEL spectrum is therefore be used to estimate the $\mathrm{sp}^{3}$ content, $\mathrm{sp}^{3} /\left(\mathrm{sp}^{2}+\mathrm{sp}^{3}\right)$, by comparison with a reference spectrum of known $\mathrm{sp}^{3}$ content collected under the same conditions [11]. Following Berger et al. [11] and McCulloch et al. [12], the $\mathrm{sp}^{3}$ fraction $f$ for a specimen of interest can be estimated using equation (1):

$$
f=1-\frac{\mathrm{sp}^{2}}{\mathrm{sp}^{2}+\mathrm{sp}^{3}}=1-\frac{I_{u \pi^{*}}}{I_{g \pi^{*}}} \frac{I_{g}(\Delta E)}{I_{u}(\Delta E)},
$$

where $I_{\mathrm{u} \pi^{*}}, I_{\mathrm{g} \pi^{*}}$ represent the respective integrated intensities under the $1 \mathrm{~s} \rightarrow \pi^{*}$ peaks for the specimen and the highly graphitic reference sample, respectively, and $I_{g}(\Delta E)$ and $I_{\mathrm{u}}(\Delta E)$ refer to the integrated intensities under the entire edge over an interval $\Delta E$. For the results reported here, an interval $\Delta E$ between $280 \mathrm{eV}$ and $350 \mathrm{eV}$ was used. All spectra were background subtracted using a $30 \mathrm{eV}$ wide region of the pre-edge EELS data and assuming a power law model. Fourier-ratio deconvolution [7] was also used to remove plural scattering effects before application of equation (1). The various $I_{u \pi^{*}}$ and $I_{\mathrm{g} \pi^{*}}$ were estimated by fitting a Gaussian function to these peaks. All of the 
aforementioned EELS analysis was performed using Gatan's Digital Micrograph software.

Energy-filtered electron diffraction (EFED) data [13-16] were collected for samples 2 and 3 (see Table 1) to extract the static structure factor $S(k)$ and radial distribution function $g(r)$ from the experimental diffracted intensity $I(k)$. Assuming the kinematical approximation, which is appropriate for electron diffraction from thin, lightelement disordered solids, these functions are related as follows,

$$
S(k)=\frac{I(k)}{N f^{2}(k)}=1+\frac{4 \pi \rho}{k} \int r(g(r)-1) \sin (k r) d r
$$

In equation (2), $k$ is the magnitude of the scattering vector, $N$ is related to the number of incident electrons, $\rho$ is the number density of the diffracting sample and $f(k)$ represents the atomic scattering factor. For the EFED technique, the quality of both $S(k)$ and $g(r)$ can be improved by rotationally averaging isotropic diffraction data and also acquiring $S(k)$ for longer exposures at larger $k$. In practice, it is advantageous to collect several overlapping diffraction patterns with increased exposure times for $I(k)$ collected at larger $k$ values. Post acquisition analysis can then be used to "splice” the patterns together to give improved $I(k)$ statistics over a wide range of $k$. For this work, location of the unknown diffraction pattern centers, removal of spurious x-ray spikes in $I(k)$ and two dimensional splicing were all performed using the a software package [17].

\section{Results and Discussion}

Preliminary inspection of TEM images and diffraction patterns showed that all films were amorphous and homogenous. No distinct features could be discerned apart from occasional defects caused by the macroparticles that made it through the filter. As a 
side note, we show a zero-loss filtered (using a $12 \mathrm{eV}$ window) high resolution image of a small macroparticle that became incorporated in the film (Fig. 4).

The EELS data revealed that all films had a high $\mathrm{sp}^{3}$ content, $\mathrm{sp}^{3} /\left(\mathrm{sp}^{2}+\mathrm{sp}^{3}\right)$, see Table 1. The \pm values shown in Table 1 represent half the difference between the minimum and maximum experimentally determined values. The results for sample 2, which were collected using different EELS systems, are consistent. Furthermore the $\mathrm{sp}^{3}$ fraction correlates with the plasmon-inferred densities and, with the exception of sample 4 , this trend follows that proposed by Ferrari et al. [9] (albeit using $m^{*}=0.87 m_{\mathrm{e}}$ ).

Three diffraction patterns covering a sufficiently wide range of scattering angles were collected for each of samples 2 and 3; however instabilities in the GIF system resulted in erroneous data for the last data set of sample 2, which was revealed by an abnormal fractional uncertainty profile. The $I(k)$ data for sample 2 was therefore produced using only two spliced diffraction patters, instead of three. All other diffraction patterns yielded fractional uncertainty profiles that followed expected Poisson statistics [17]. $S(k)$ and $g(r)$ for samples 2 and 3 were extracted from equation (2) using the approximate densities listed in Table 1 and $f(k)$ given by Kirkland [18]. Small constants were subtracted from $I(k)$ to correct for errors in dark count estimates, which were determined by ensuring that $S(k)$ oscillate about the value one for large $k$. For calculation of $g(r)$ using equation 2, it is conventional to use damping functions to limit Fourier truncation errors, which produce unphysical oscillations in $g(r)$ for small values of $r$ [19]. Such functions were not employed here however, since $I(k)$ was measured over a sufficiently large range of $k$ values for both samples. Before inversion of equation 2 , the $S(k)$ data were truncated to $k_{\max }=24 \AA^{-1}$ and $k_{\max }=27 \AA^{-1}$ for samples 2 and 3 
respectively. These data ranges were chosen so that the fractional uncertainty in $S(k)$ was no greater than $40 \%$. Some spurious ripples in $g(r)$ for small $r$ remained, which were set to zero to produce the plots shown in figure 5 . For consistency, the $g(r)$ values in figure 5 were used to recalculate each $S(k)$ via equation (2), which are plotted in figure 6 .

Figures 6 indicates that there is practically no difference between the $S(k)$ values for samples 2 and 3, which were created using plasma and substrate biasing, respectively, with all other parameters unchanged. Some spurious ripples in the $g(r)$ of sample 2 are evident in figure 5, which resulted from truncation of $I(k)$ at lower $k$ caused by the use of only two splices. However both $g(r)$ functions are quite similar, with a first nearest neighbor peak at $r=1.50 \AA$, which is close to the diamond value. Figures 5 and 6 indicate that the inter-atomic pair correlated structures for samples 2 and 3 are practically the same.

From the data of Table 1 it is apparent that the $300 \mathrm{~V}$ bias resulted in a higher $\mathrm{sp}^{3}$ content than the $120 \mathrm{~V}$, which is somewhat surprising since it is generally known that carbon ion energies of about $100-200 \mathrm{eV}$ lead to the highest $\mathrm{sp}^{3}$ content. The formation of diamond bonds is facilitated by densification via energetic condensation, i.e. insertion of carbon ions in the surface and subsurface layers. At energies exceeding some $100 \mathrm{eV}$, thermal spike annealing relaxes the stressed material and shifts the $\mathrm{sp}^{3} / \mathrm{sp}^{2}$ ratio to lower values [20]. Therefore, the results reported here may be affected by the limited current capabilities of the bias pulser: When the fluctuating ion current approaches the limiting value of the pulser, the voltage may be lower than the nominal value. Using the voltage probe, we have occasionally observed such reduced bias voltage (e.g. down to $50 \mathrm{~V}$ for 
the $120 \mathrm{~V}$ nominal voltage), which shifts the effective bias closer to values typical for taC films of maximum $\mathrm{sp}^{3}$ content, hardness and compressive stress.

\section{Conclusions}

The results show that ta-C films with high $\mathrm{sp}^{3}$ content can be obtained when the substrate is grounded and the ion energy is determined by plasma biasing. The films obtained this way are comparable to films obtained by the more conventional substrate biasing. In particular, the similar data for our films 2 (plasma bias) and 3 (substrate bias) prove that point.

We focused on ta-C films for this experiment on plasma bias because the wellcharacterized diamond-like nature of this material acts as a "fingerprint" for the energetics of the condensing species. Therefore, bias effects are especially important for carbon films. The plasma bias principle is not limited to carbon plasmas may also be applied to other material systems.

\section{Acknowledgements}

N. Pasaja was supported by the Royal Golden Jubilee Ph.D. Program, the Thailand Research Fund, Thailand. S.H.N. Lim acknowledges funding by the American Australia Association for the 2004 ANZ Fellowship. This work was supported by the Assistant Secretary for Energy Efficiency and Renewable Energy, Office of Building Technology,

of the U.S. Department of Energy under Contract No. DE-AC02-05CH11231. 


\section{References}

[1] A. Anders, (Ed.), Handbook of Plasma Immersion Ion Implantation and Deposition. New York: John Wiley \& Sons, 2000.

[2] R. A. MacGill, M. R. Dickinson, A. Anders, O. R. Monteiro, and I. G. Brown, Rev. Sci. Instrum. 69 (1998) 801.

[3] A. Anders, I. G. Brown, R. A. MacGill, and M. R. Dickinson, J. Phys. D: Appl. Phys. 31 (1998) 584.

[4] A. Anders, Surf. Coat. Technol. 120-121 (1999) 319.

[5] M. M. M. Bilek and A. Anders, Plasma Sources Sci. Technol. 8 (1999) 488.

[6] D. G. McCulloch, E. G. Gerstner, D. R. McKenzie, S. Prawer, and R. Kalish, Phys. Rev. B 52 (1995) 850-857.

[7] R. F. Egerton, Electron Energy-Loss Spectroscopy in the Electron Microscope, 2nd ed., New York: Plenum Press, 1996.

[8] D. R. McKenzie, Rep. Prog. Phys. 59 (1996) 1611.

[9] A. C. Ferrari, A. Libassi, B. K. Tanner, V. Stolojan, J. Yuan, L. M. Brown, S. E. Rodil, B. Kleinsorge, and J. Robertson, Phys. Rev. B 62 (2000) 11089-11103.

[10] P. H. Gaskell, A. Saeed, P. Chieux, and D. R. McKenzie, Phys. Rev. Lett. 67 (1991) 1286-1289.

[11] S. D. Berger, D. R. McKenzie, and P. J. Martin, Phil. Mag. Lett. 57 (1988) 285.

[12] D. G. McCulloch, S. Prawer, and A. Hoffman, Phys. Rev. B 50 (1994) 59055917.

[13] D. J. H. Cockayne, D. R. McKenzie, and D.A. Muller, Microsc. Microanal. Microstruct. 2 (1991) 359. 
[14] L. C. Qin and L. W. Hobbs, Journal of Non-Crystalline Solids 192-193 (1995) 456.

[15] D. Heinemann and W. Mader, Ultramicroscopy 74 (1998) 113.

[16] T. Petersen, I. Yarovsky, I. Snook, D. G. McCulloch, and G. Opletal, Carbon 42 (2004) 2457.

[17] T. C. Petersen, W. McBride, D. G. McCulloch, I. K. Snook, and I. Yarovsky, Ultramicroscopy 103 (2005) 275; (beta version of the software is available online at www.rmit.edu.au/physics/efdp)

[18] E. J. Kirkland, Advanced Computing in Electron Microscopy. New York: Kluwer Academic Publishers Group, 1998.

[19] R. E. Franklin, Acta Cryst. 3 (1950) 107.

[20] M. M. M. Bilek and D. R. McKenzie, Surf. Coat. Technol. 200 (2006) 4345. 


\section{Figure Captions}

Fig. 1 (a) Setup of pulsed cathodic arc source with $90^{\circ}$ open coil filter; the anode was grounded and the substrate was negatively pulsed biased; (b) Setup for plasma biasing: the substrate is grounded whereas the reference potential of the arc and filter power supply can be shifted positively by a pulsed plasma bias supply.

Fig. 2 Pulse-forming network (PFN) circuit supplying power to the pulsed arc as used in our plasma biasing experiment. A straightforward approach to shift the reference potential involves operating the charging supply via an isolation transformer. Here it is indicated that inductive isolation can be used, provided the bias pulses are sufficiently short.

Fig. 3 Example of arc current (100 A/div), plasma bias voltage (100 V/div), and plasma bias current ( $1 \mathrm{~A} / \mathrm{div})$ as a function of time $(50 \mu \mathrm{s} / \mathrm{div})$. To better visualize the bias pulses, not all of the arc pulse length is shown. One can see that the bias current appears with some delay, which is associated with the time-of-flight of the plasma from the cathode to the substrate. The initial peaks in the bias current are due to the capacitive load of cable and sheath. The initial negative dip in the bias voltage is driven by the electrons of the pulsed plasma edge.

Fig. 4 Energy-filtered TEM image (with a zero loss window of $12 \mathrm{eV}$ ) of a macroparticle embedded in ta-C film. The film itself is featureless but the macroparticle exhibit curved graphitic planes (image obtained using a Phillips CM120 TEM). 
Fig. 5 Radial distribution functions $g(r)$ for samples 2 (plasma bias) and 3 (substrate bias)

Fig. 6 Static structure factors $S(k)$ for samples 2 (plasma bias) and 3 (substrate bias). 


\section{Table and Table Caption}

Table 1. Compilation of data for ta-C films deposited by plasma biasing. For comparison, data for a sample made with conventional biasing and at floating potential were added.

\begin{tabular}{|c|c|c|c|c|c|}
\hline $\begin{array}{l}\text { Sample } \\
\#\end{array}$ & $\begin{array}{l}\text { Pulsed Bias } \\
\text { (V) }\end{array}$ & $\begin{array}{l}\text { Film } \\
\text { thickness } \\
(\mathrm{nm})\end{array}$ & $\mathrm{sp}^{3} /\left(\mathrm{sp}^{3}+\mathrm{sp}^{2}\right)$ & $\begin{array}{l}\text { Density } \\
\left(\mathrm{g} / \mathrm{cm}^{3}\right)\end{array}$ & $\begin{array}{l}\text { Plasmon } \\
\text { Energy (eV) }\end{array}$ \\
\hline 1 & $\begin{array}{l}300 \\
\text { Plasma bias }\end{array}$ & 35 & $0.64 \pm 0.02$ & 3.28 & $30.1 \pm 0.2$ \\
\hline 2 & $\begin{array}{l}300 \\
\text { Plasma bias }\end{array}$ & 55 & $0.65 \pm 0.04$ & 3.28 & $30.1 \pm 0.6$ \\
\hline $2 *$ & $\begin{array}{l}300 \\
\text { Plasma bias }\end{array}$ & 55 & $0.69 \pm 0.02$ & 3.36 & $30.5 \pm 0.3$ \\
\hline 3 & $\begin{array}{l}300 \\
\text { Substrate bias }\end{array}$ & 55 & $0.60 \pm 0.07$ & 3.15 & $29.5 \pm 0.5$ \\
\hline 4 & $\begin{array}{l}120 \\
\text { Plasma bias }\end{array}$ & 55 & $0.51 \pm 0.03$ & 2.65 & $27.1 \pm 1.3$ \\
\hline 5 & $\begin{array}{l}0 \text { plasma bias, } \\
\text { Substrate at } \\
\text { ground }\end{array}$ & 55 & $0.47 \pm 0.07$ & 2.74 & $27.5 \pm 0.6$ \\
\hline 6 & $\begin{array}{l}0 \text { plasma bias, } \\
\text { Substrate } \\
\text { floating }\end{array}$ & 55 & $0.64 \pm 0.09$ & 3.14 & $29.5 \pm 0.5$ \\
\hline
\end{tabular}

* Results obtained using the VGSTEM. 


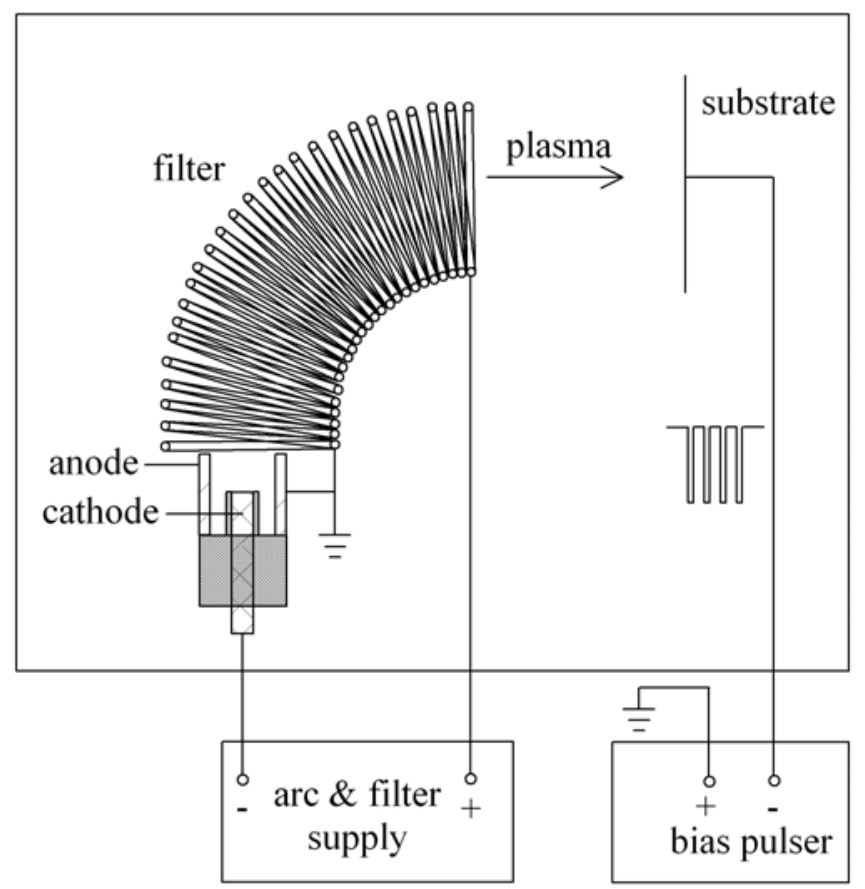

Fig. 1(a) 


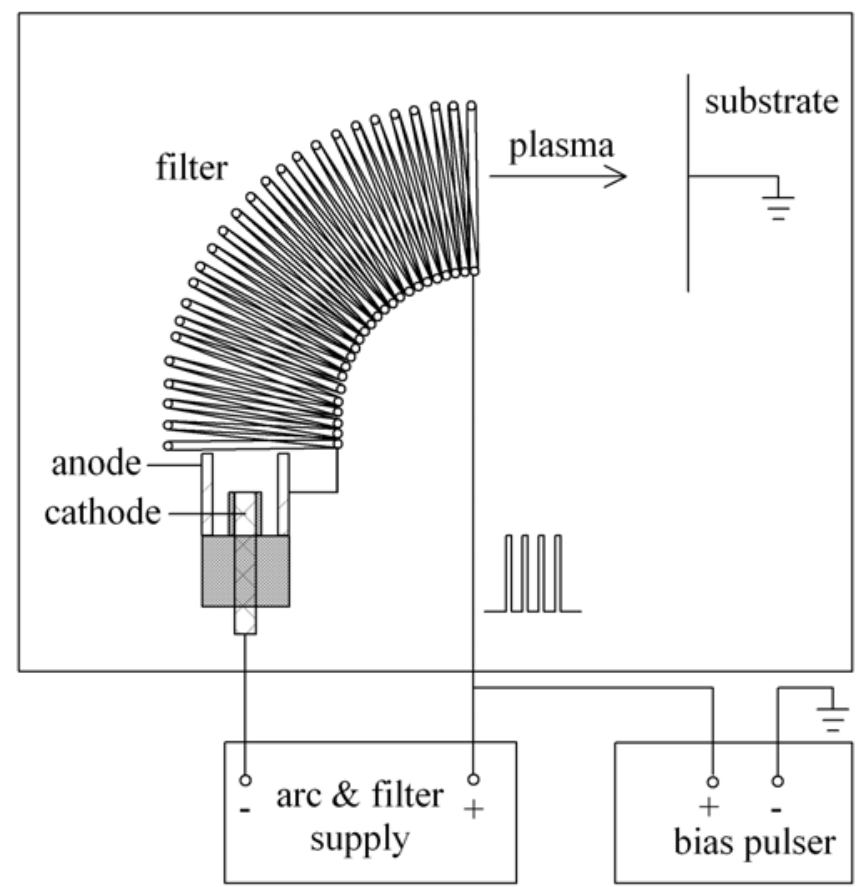

Fig. 1(b) 


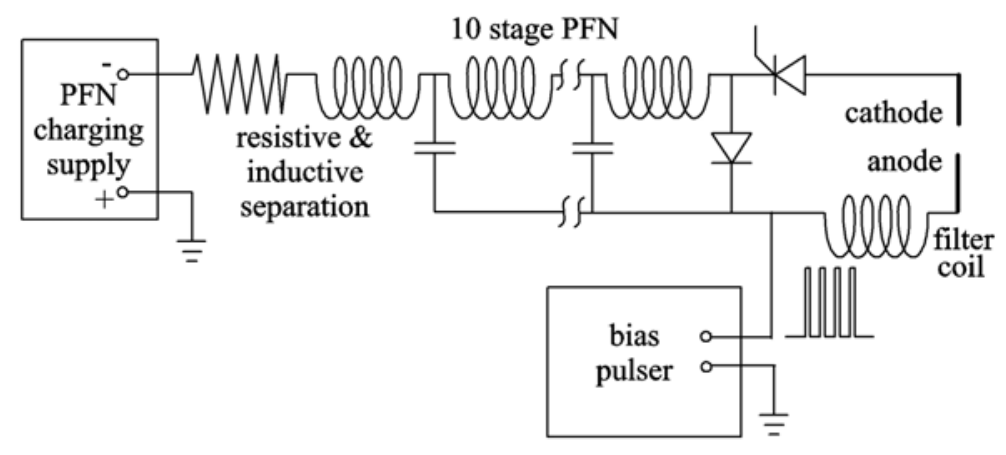

Fig. 2 


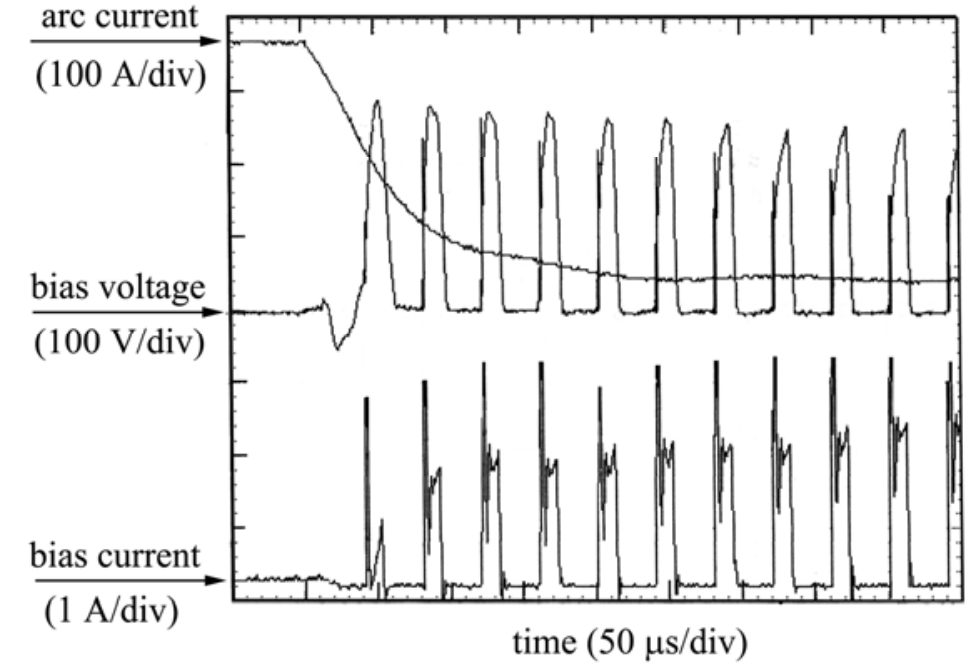

Fig. 3 


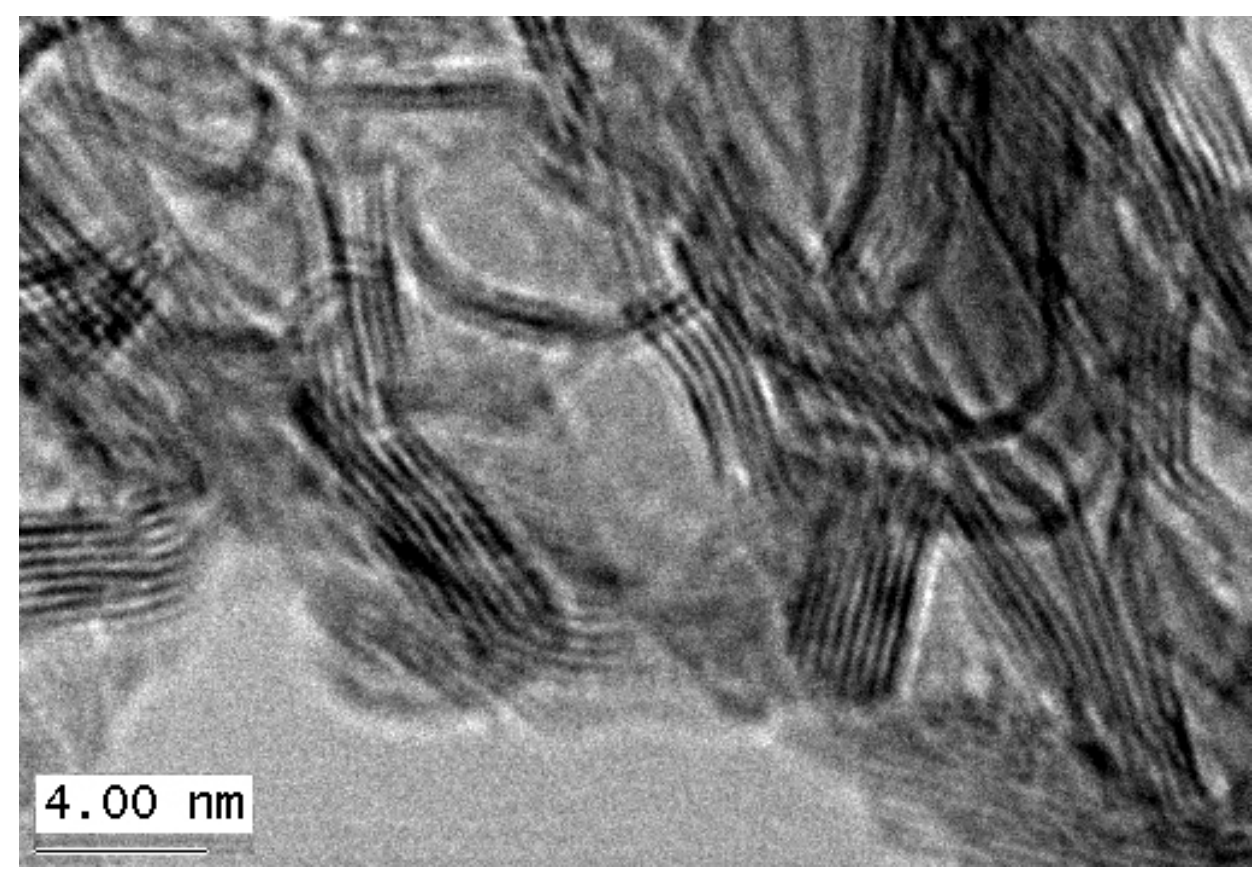

Fig. 4 


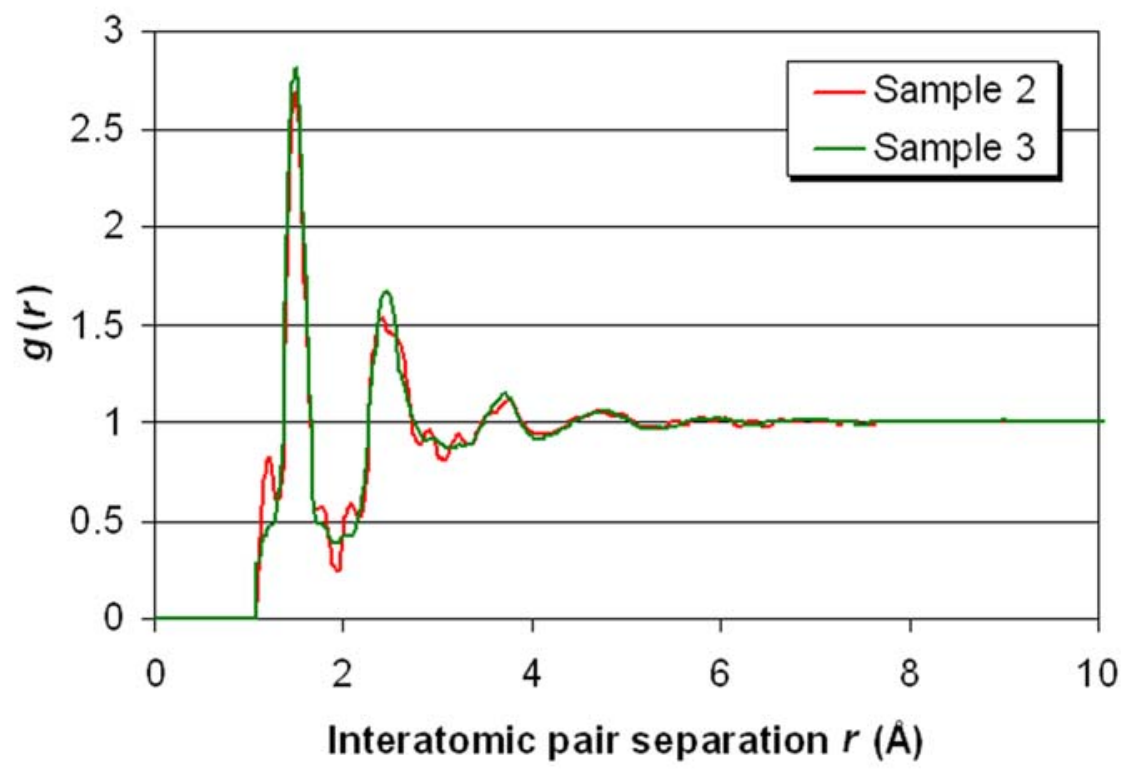

Fig. 5 


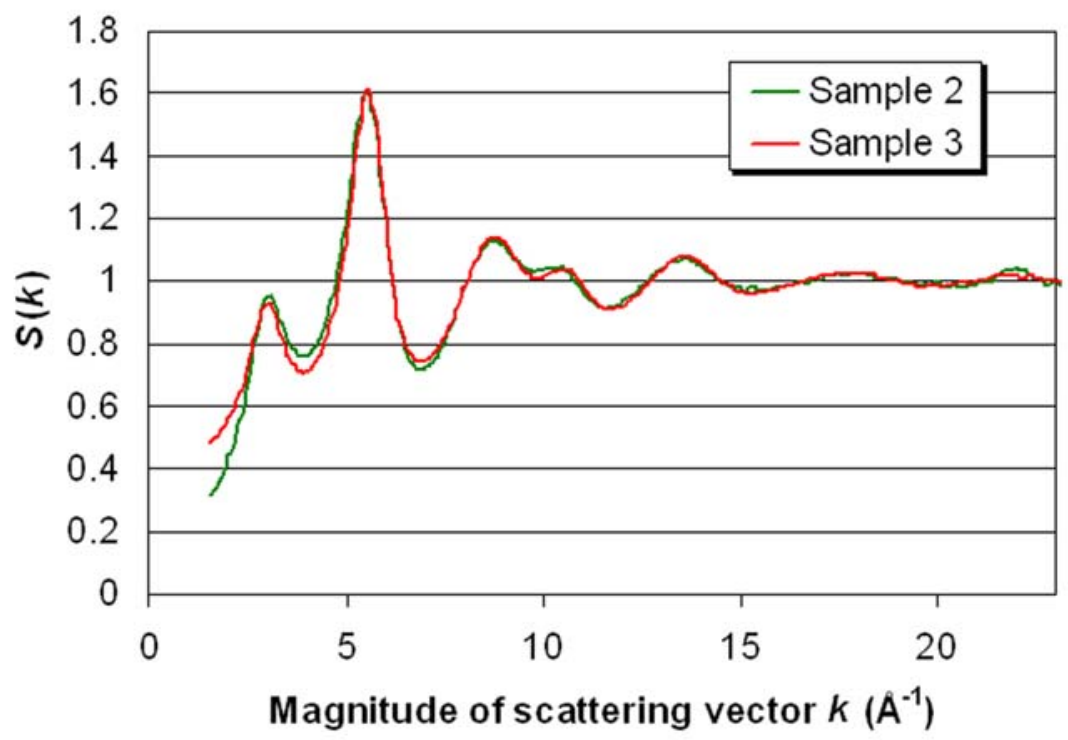

Fig. 6 